\title{
Immunocytochemical localization of vasotocin-like immunoreactivity in the brain of the cartilaginous fish, Scyliorhinus caniculus
}

Mauro Vallarino, Carla Viglietti-Panzica, and Gian Carlo Panzica

Cell Tissue Res (1990) 262:507-513

According to our Thesaurus for Animal Index (see General Index: Vols. 201-250, 1988), based on representative taxonomic manuals, the name of the species examined in this study should have been Scyliorhinus caniculus throughout the paper. It is correct in the title. 